\title{
LETTER \\ A Study of Qualitative Knowledge-Based Exploration for Continuous Deep Reinforcement Learning
}

\author{
Chenxi $\mathrm{LI}^{\dagger \text { a) }}$, Student Member, Lei CAO ${ }^{\dagger}$, Xiaoming $\mathrm{LIU}^{\dagger}$, Xiliang $\mathrm{CHEN}^{\dagger}$, Zhixiong XU $\mathrm{X}^{\dagger}$, \\ and Yongliang ZHANG ${ }^{\dagger}$, Nonmembers
}

\begin{abstract}
SUMMARY As an important method to solve sequential decisionmaking problems, reinforcement learning learns the policy of tasks through the interaction with environment. But it has difficulties scaling to largescale problems. One of the reasons is the exploration and exploitation dilemma which may lead to inefficient learning. We present an approach that addresses this shortcoming by introducing qualitative knowledge into reinforcement learning using cloud control systems to represent 'if-then' rules. We use it as the heuristics exploration strategy to guide the action selection in deep reinforcement learning. Empirical evaluation results show that our approach can make significant improvement in the learning process.

key words: deep reinforcement learning, knowledge, exploration strategy, cloud control systems
\end{abstract}

\section{Introduction}

Reinforcement learning (RL) [1] is a promising approach to create intelligent systems that adopts a mechanism of "trial and error" to interact with the unknown environment, in order to learn the policy of the task. RL requires much less labeled samples than supervised learning, but leads to a huge number of training episodes to a good solution. This problem gets worse in high dimensional and continuous tasks.

One effective method for scaling RL is to make use of human knowledge as exploration strategy. Knowledge, which contains experience, values, cognitive rules and expert opinions, can be used to improve the learning efficiency. The approach has proven effective in a number of domains. Celiberto et al. [2] proposed a heuristic exploration function through case-based reasoning and Bianchi et al. [3] transferred existing similar cases or trajectories to heuristics. Bianchi et al. [4] also proved the convergence of heuristic exploration and the boundary of error estimation in theoretical analysis. Besides, Santo et al. [5] proposed Dyna-H algorithm based on Dyna-Q framework, using the A* algorithm as a heuristic function, providing guidance for the planning part of the algorithm. Miyazaki [6] proposed the k-certainty exploration method which builds a maximum likelihood model of the state transition probabilities to guide action selection.

Manuscript received May 19, 2017.

Manuscript revised June 27, 2017.

Manuscript publicized July 26, 2017.

$\dagger$ The authors are with the Institute of Command Information System, PLA University of Science and Technology, Nanjing, 210007 China.

a)E-mail: streamorning@qq.com

DOI: 10.1587/transinf.2017EDL8112
In addition to case-based knowledge and experience obtained in the learning process, priori knowledge, mainly in the form of 'if-then' rules, is also an important part of them. Kuhlmann et al. [7] mapped advice expressed in natural language to precise 'if-then' rules, then provided them to a reinforcement learner in the task of RoboCup Soccer. The supervised reinforcement learning (SRL) framework proposed by Moreno et al. [8] is an amalgamation of $Q$ value and prior knowledge sources. The final action is decided by control module according to credit assignment. We should notice that all the works above are mainly based on the use of precise quantitative rules. For example, in the RoboCup Soccer [7], one piece of advice called 'Hold Advice', suggested that the player in possession of the ball should hold onto the ball rather than pass it if no opponents are within $8 \mathrm{~m}$. If $8.1 \mathrm{~m}$, the advice may change different.

In many task domains, knowledge or rules are often described by qualitative and imprecise words such as 'fast', 'long', rather than the quantitative values. Therefore, we present a new method using cloud control systems to represent the uncertainty rules, providing advice to deep reinforcement learning as heuristic exploration strategy.

We carried out our experiments on OpenAI Gym [9] with the environments of 'InvertedPendulum-v1' and 'LunarLanderContinuous-v2'. Both of them have high dimensional state spaces and continuous action spaces. We present empirical evidences that our approach can make effective use of qualitative rules and significantly reduce the number of training episodes.

\section{Qualitative Knowledge-Based Exploration}

Based on the membership function in fuzzy theory [10], Li et al. [11] proposed a concept of membership clouds to represent linguistic terms. The essence of the cloud model is to add the randomness to the standard deviation of the membership function, integrating fuzziness and randomness in an inseparable way. Then they proposed the algorithm of cloud reasoning and cloud control systems [12]. In this work, we present the representation and deduction of cloud control systems in theoretical analysis, and then we use it to represent the qualitative rules, providing advice to deep reinforcement learning. 


\subsection{Cloud Control}

Cloud control systems consists of cloud model, singleton fuzzifier, center average defuzzifier and product inference engine. The fuzzifier is defined as a mapping from a realvalued point to a fuzzy set. Singleton fuzzifier (1) and Mamdani product inference engine (2) are of the following form:

$$
\begin{aligned}
& \mu_{\mathrm{A}^{\prime}}(s)= \begin{cases}1 & s=s^{*} \\
0 & \text { others }\end{cases} \\
& \mu_{B^{\prime}}(a)=\max _{l=1}^{M}\left[\sup _{x \in U}\left(\mu_{A^{\prime}}(s) \prod_{i=1}^{n} \mu_{A, i, l}\left(s_{i}\right) \mu_{B, l}(a)\right)\right]
\end{aligned}
$$

where $s$ is n-dimensional vector representing the input state, $i=1,2, \ldots, n$. $a$ is the output action. $M$ is the number of rules in the qualitative rule base, $l=1,2, \ldots, M . \mu_{A, i, l}\left(s_{i}\right)$ is a cloud model producing drops under a given numerical value $s_{i}$ in rule $l$, we can call it state-cloud. $\mu_{B, l}(a)$ is a cloud model under the condition of action $a$ in rule $l$, we call it action-cloud. Center average defuzzifier determines $a^{*}$ as

$$
a^{*}=\sum_{l=1}^{M} \bar{a}_{B, l} \omega_{l} / \sum_{l=1}^{M} \omega_{l}
$$

where $\bar{a}_{B, l}$ is the center of $l^{\prime} t h$ action-cloud, and $\omega_{l}$ is the height of $l^{\prime}$ th action-cloud.

Substituting (1) into (2), we have

$$
\mu_{B^{\prime}}(a)=\max _{l=1}^{M}\left[\prod_{i=1}^{n} \mu_{A, i, l}\left(s_{i}^{*}\right) \mu_{B, l}(a)\right]
$$

The center of $\prod_{i=1}^{n} \mu_{A, i, l}\left(s_{i}^{*}\right) \mu_{B, l}(a)$ is also the center of $\mu_{B, l}(a)$, thus

$$
\bar{a}_{B, l}=E x_{B_{l}}
$$

And the height of $\mu_{B, l}(a)$ is 1 , then

$$
\omega_{l}=\prod_{i=1}^{n} \mu_{A, i, l}\left(s_{i}^{*}\right)
$$

Substituting (5) and (6) into (3), we obtain the action

$$
a^{*}=\sum_{l=1}^{M} E x_{B_{l}}\left(\prod_{i=1}^{n} \mu_{A, i, l}\left(s_{i}{ }^{*}\right)\right) / \sum_{l=1}^{M}\left(\prod_{i=1}^{n} \mu_{A, i, l}\left(s_{i}{ }^{*}\right)\right)
$$

where state-cloud $\mu_{A, i, l}\left(s_{i}^{*}\right)$ :

$$
\mu_{A, i, l}\left(s_{i}^{*}\right)=\mathrm{e}^{-\left(\frac{s_{i}-E x_{A, l, i}}{\left.{\operatorname{Norm}\left(E n_{A, l, i}, H\right.}_{A, l, i}\right)}\right)^{2}}
$$

and action-cloud $\mu_{B, l}(a)$ :

$$
\mu_{B, l}(a)=\mathrm{e}^{-\left(\frac{a-E x_{\mathrm{B}, l}}{N o r m\left(E n_{\mathrm{B}, l}, H e_{\mathrm{B}, l}\right)}\right)^{2}}
$$

where $\operatorname{Norm}\left(E n_{A, l, i}, H e_{A, l, i}\right)$ and $\operatorname{Norm}\left(E n_{\mathrm{B}, l}, H e_{\mathrm{B}, l}\right)$ are the normal random numbers.

Referring to the theory of fuzzy control [9], we can easily prove that cloud control systems can approximate any nonlinear function to arbitrary accuracy by substituting (7) into Stone-Weierstrass theorem.

\subsection{Qualitative Knowledge-Based Exploration}

Sometimes it is difficult to directly find good cloud systems or fuzzy systems for the particular applications. In this work, we present a framework using cloud control systems to represent the qualitative rules, providing advice to deep reinforcement learning as heuristic exploration strategy. With the guidance of cloud control, the agent can improve the learning efficiency. On the other hand, we do not need a set of complete rules and accuracy parameters of cloud control systems with the help of reinforcement learning.

The framework of cloud control and deep reinforcement learning is shown in Fig. 1, that comprises several basic modules: the RL agent, the cloud controller, and the $\varepsilon$ controller module. We call the action given by cloud controller as advice action. And we call the action proposed by RL agent with noise exploration as noise action. In each step, $\varepsilon$-controller module selects the final action according to the two actions. The details can be described below:

a) If cloud controller cannot provide advice action since no rules are enabled to fit current state, $\varepsilon$-controller should adopt noise action.

b) If cloud controller and RL module can provide action, $\varepsilon$-controller will adopt advice action with the probability of $\varepsilon$ and adopt noise action with the probability of $1-\varepsilon$ as selected action.

c) $\varepsilon$ decreases at a rate of $\Delta \varepsilon$ in the learning process. We evaluate the performance of cloud controller (denoted by $P_{\varepsilon}$ ) first. In the reinforcement learning process, the learned policy will be tested without noise action or advice action by every $N$ training episode. If the evaluation score is lower than $P_{\varepsilon}, \Delta \varepsilon$ (denoted by $\Delta_{\text {before }} \varepsilon$ ) can stay be lower to encourage the adoption of advice action. After the evaluation score becomes higher than $P_{\varepsilon}, \Delta \varepsilon$ (denoted by $\Delta_{a f t e r} \varepsilon$ ) should become higher to restrain advice action.

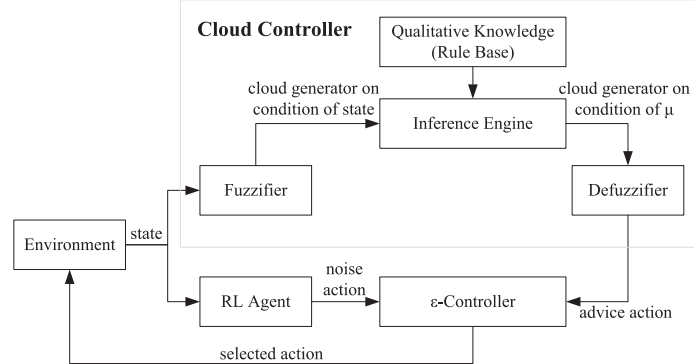

Fig. 1 Framework of cloud control system and deep reinforcement learning. 


\section{Experimental Result}

We carried out our experiments on OpenAI Gym [9] with continuous environments of 'InvertedPendulum-v1' and 'LunarLanderContinuous-v2'.

'InvertedPendulum-v1' environment is simulated in 'MuJoCo' physics engine including a pendulum and a cart. The goal of this task is to prevent the pendulum from falling over through applying a continuous force to the cart. 'LunarLanderContinuous-v2' is a video game to control a lander to land on the surface of moon safely which is based on a 2D physics engine called 'Box2d'. More details can be found in homepage or in source code of OpenAI Gym. We only used the low dimensional feature vectors rather than the pixel features. Table 1 lists the state and action dimensionality of the environments and the maximum steps in each episode.

We used novel DDPG [13] as the basic RL algorithm and used the same parameters as the original paper. In $\varepsilon$ controller module, $\varepsilon$ was initialized by 0.999 with $\Delta_{\text {before }} \varepsilon=$ $10^{-6}$ and $\Delta_{\text {after }} \varepsilon=10^{-4}$.

We independently executed each method 10 times respectively on every task. For each running time, the learned policy will be tested 10 episodes respectively without exploration noise or prior knowledge by every 10 training episodes to calculate the average scores. We report the mean and standard deviation of the convergent episodes number and the scores of the best policy.

\subsection{InvertedPendulum-v1}

For this task, we can get the qualitative control rules based on the common sense of how to balance the inverted pendulum:

a) IF $\theta$ deflects substantially (backward) THEN apply more force to the cart (backward)

b) IF $\theta$ deflects slightly (backward) THEN apply normal force to the cart (backward)

c) IF $\theta$ is zero THEN apply none

d) IF $\theta$ deflects slightly (forward) THEN apply normal force to the cart (forward)

e) IF $\theta$ deflects substantially (forward) THEN apply more force to the cart (forward)
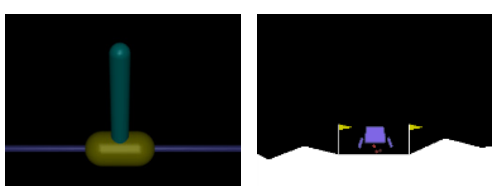

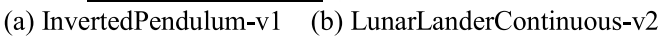

Fig. 2 Example screenshots of environments

Table 1 Dimensionality of the environments.

\begin{tabular}{lccc}
\hline Task name & $\operatorname{dim}(\mathrm{s})$ & $\operatorname{dim}(\mathrm{a})$ & Max step \\
\hline InvertedPendulum-v1 & 4 & 1 & 1000 \\
LunarLanderContinuous-v2 & 8 & 2 & 1000 \\
\hline
\end{tabular}

where the angle of pendulum is denoted by $\theta$.

Using state-clouds and action-clouds to represent the qualitative rules described above, parameters are shown in Table 2. Each cloud contains $(E x, E n, H e)$. Parameters were set directly by experience without any adjustment and correctness.

We compared cloud control and fuzzy control without reinforcement learning module. Results are shown in Table 3. We used random control as baseline. It can be found that cloud control and fuzzy control systems can both reach about 200 points, much higher than the baseline, but much lower than the highest score of 1000 points. Then we used these controllers to provide advice to DDPG to accelerate learning process. We set $P_{\varepsilon}$ as 180 points in $\varepsilon$-controller module. Table 4 shows the performance of each algorithm. All of them could learn the best policy with highest score, but the convergent episodes number differs greatly. DDPG requires 2464 episodes, while DDPG with fuzzy control requires 675, and DDPG with cloud control only requires 470 .

\subsection{LunarLanderContinuous-v2}

In 'LunarLanderContinuous-v2', state vector consist of 8 features which we used only 4 of them in cloud control and fuzzy control systems, these are: the horizontal and vertical coordinates $s_{1}$ and $s_{2}$, the vertical velocity $s_{4}$ and the angle $s_{5}$. Action is two real values vector. First value controls vertical engine (denoted by $a_{1}$ ), and the second value controls horizontal engine (denoted by $a_{2}$ ).

For vertical engine $a_{1}$, we can intuitively guess that when $s_{2}$ and $s_{4}$ become lower, $a_{1}$ should be greater. For horizontal engine $a_{2}$, when $s_{1}$ becomes lower (i.e. the left offset become greater) and $s_{5}$ is higher (positive angle), $a_{2}$ should be lower (i.e. the left engine becomes greater). For simplicity, we combine the two variables into a single variable respectively:

$$
\begin{aligned}
& s_{v}=c_{v, 1} \cdot s_{2}+c_{v, 2} \cdot s_{4} \\
& s_{h}=c_{\mathrm{h}, 1} \cdot s_{1}-c_{\mathrm{h}, 2} \cdot s_{5}
\end{aligned}
$$

We simply set these parameters $c_{v, 1}, c_{v, 2}, c_{\mathrm{h}, 1}, c_{\mathrm{h}, 2}=0.5$.

Table 2 Parameters of the state-cloud and action-cloud.

\begin{tabular}{ccc}
\hline Rules & State-cloud & Action-cloud \\
\hline a & $(-1,0.13,0.013)$ & $(-1,0.15,0.013)$ \\
b & $(-0.5,0.1,0.005)$ & $(-0.5,0.1,0.005)$ \\
c & $(0,0.05,0.01)$ & $(0,0.05,0.01)$ \\
d & $(0.5,0.1,0.005)$ & $(0.5,0.1,0.005)$ \\
e & $(1,0.13,0.013)$ & $(1,0.15,0.013)$ \\
\hline
\end{tabular}

Table 3 Scores of random control, fuzzy control and cloud control.

\begin{tabular}{lccc}
\hline Results & random control & fuzzy control & cloud control \\
\hline Scores & $8.34 \pm 4.6$ & $195.56 \pm 7.6$ & $200.16 \pm 9.1$ \\
\hline
\end{tabular}

Table 4 Performance of DDPG, DDPG with fuzzy and cloud control.

\begin{tabular}{lccc}
\hline $\begin{array}{l}\text { Running } \\
\text { times }\end{array}$ & DDPG & $\begin{array}{c}\text { DDPG with } \\
\text { fuzzy control }\end{array}$ & $\begin{array}{c}\text { DDPG with } \\
\text { cloud control }\end{array}$ \\
\hline Episodes & $2464 \pm 248.7$ & $675 \pm 151.0$ & $\mathbf{4 7 0} \pm \mathbf{6 9 . 8}$ \\
\hline Scores & $1000 \pm 0$ & $1000 \pm 0$ & $1000 \pm 0$ \\
\hline
\end{tabular}


Thus we can conclude these rules as: for vertical engine,

a) IF $s_{v}$ becomes lower THEN $a_{1}$ should be greater

b) IF $s_{v}$ changes slightly THEN $a_{1}$ should be normal

c) IF $s_{v}$ becomes higher THEN $a_{1}$ should be lower and for horizontal engine,

a) IF $s_{h}$ becomes lower THEN $a_{2}$ should be lower

b) IF $s_{h}$ changes slightly THEN $a_{2}$ should be normal

c) IF $s_{h}$ becomes higher THEN $a_{2}$ should be greater

Parameters are shown in Table 5 and Table 6.

According to the scores shown in Table 7, cloud control and fuzzy control without DDPG reach 180 points, and the policy is still unstable (high standard deviation). We set $P_{\varepsilon}$ as 180 points in ' $\varepsilon$-Controller' module. Final results are shown in Table 8. It can be found that DDPG needs 12310 episodes to reach convergence, while DDPG with fuzzy control needs 2500, DDPG with cloud control needs 2637. And the learned policy of the last two algorithms is much stable (low standard deviation) than DDPG. Figure 3 is the performance curve of each algorithm. We present em-

Table 5 Parameters of cloud and fuzzy control for vertical engine.

\begin{tabular}{ccccc}
\hline \multirow{2}{*}{ Rules } & \multicolumn{2}{c}{ Condition } & \multicolumn{2}{c}{ Action } \\
& cloud & fuzzy & cloud & fuzzy \\
\hline a & $(-1,0.3,0.008)$ & $(-1,0.3)$ & $(-1,0.02,0.004)$ & $(-1,0.02)$ \\
b & $(-0.07,0.015,0.002)$ & $(-0.07,0.015)$ & $(-0.5,0.2,0.001)$ & $(-0.5,0.2)$ \\
c & $(1,0.4,0.008)$ & $(1,0.4)$ & $(1,0.32,0.01)$ & $(1,0.32)$ \\
\hline
\end{tabular}

Table 6 Parameters of cloud and fuzzy control for horizontal engine.

\begin{tabular}{ccccc}
\hline \multirow{2}{*}{ Rules } & \multicolumn{2}{c}{ Condition } & \multicolumn{2}{c}{ Action } \\
& cloud & fuzzy & cloud & fuzzy \\
\hline a & $(-1,0.28,0.02)$ & $(-1,0.28)$ & $(-1,0.1,0.002)$ & $(-1,0.1)$ \\
b & $(0,0.04,0.005)$ & $(0,0.04)$ & $(0,0.3,0.01)$ & $(0,0.3)$ \\
c & $(1,0.28,0.02)$ & $(1,0.28)$ & $(1,0.1,0.002)$ & $(1,0.1)$ \\
\hline
\end{tabular}

Table 7 Scores of random control, fuzzy control and cloud control.

\begin{tabular}{lccc}
\hline Results & random control & fuzzy control & cloud control \\
\hline Scores & $-760.78 \pm 159.6$ & $182.59 \pm 91.6$ & $187.98 \pm 86.4$ \\
\hline
\end{tabular}

Table 8 Performance of DDPG, DDPG with fuzzy and cloud control.

\begin{tabular}{lccc}
\hline Running times & DDPG & $\begin{array}{c}\text { DDPG with fuzzy } \\
\text { control }\end{array}$ & $\begin{array}{c}\text { DDPG with cloud } \\
\text { control }\end{array}$ \\
\hline Episodes & $12310 \pm 3295.8$ & $\mathbf{2 5 0 0} \pm \mathbf{4 5 6 . 6}$ & $2637 \pm 454.9$ \\
\hline Scores & $215.24 \pm 52.7$ & $232.24 \pm 17.9$ & $\mathbf{2 3 5 . 2 9} \pm \mathbf{2 1 . 6}$ \\
\hline
\end{tabular}

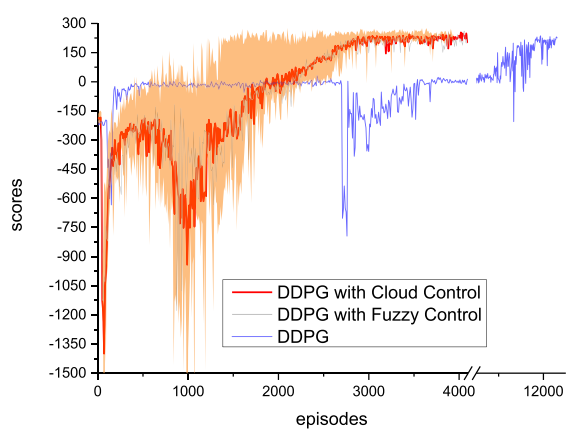

Fig. 3 The performance of each algorithm. The solid line represents the average scores of each algorithm, and the orange shaded area shows the two extreme values of DDPG with cloud control. pirical results that our approach can make effective use of qualitative rules, improve policy stability and significantly reduce the number of training episodes.

\section{Conclusion}

We believe that advice-taking methods are critical for scaling reinforcement-learning methods to large-scale problems. This paper proposes a heuristic exploration strategy based on cloud control systems to represent these rules. In the learning process, the qualitative rules are used to guide the action selection, so that the agent can quickly learn the effective policy and avoid the blindness of the exploration. In contrast to the direct use of cloud control or fuzzy control systems, the proposed algorithm uses advice as guidance in reinforcement learning. So it does not need the accuracy parameters of initial control model and a set of complete rules. In complex problems, this algorithm may be more practical.

\section{References}

[1] R. Sutton and A. Barto, Reinforcement Learning: An Introduction, MIT Press, 1998.

[2] L.A. Celiberto, J.P. Matsuura, R.L.D. Mantaras, and R.A.C. Bianchi, Using Cases as Heuristics in Reinforcement Learning: A Transfer Learning Application, IJCAI, pp.1211-1217, 2011.

[3] R.A.C. Bianchi, L.A. Celiberto, P.E. Santos, J.P. Matsuura, and R.L. de Mantaras, Transferring knowledge as heuristics in reinforcement learning: A case-based approach, Artificial Intelligence, vol.226, pp.102-121, 2015.

[4] R.A.C. Bianchi, C.H.C. Ribeiro, and A.H.R. Costa, Heuristically Accelerated Reinforcement Learning: Theoretical and Experimental Results, Frontiers in Artificial Intelligence \& Applications, p.242, 2012.

[5] M. Santos, J.A. Martín H, V. López, and G. Botella, Dyna-H: A heuristic planning reinforcement learning algorithm applied to roleplaying game strategy decision systems, Knowledge-Based Systems, vol.32, no.8, pp.28-36, 2012.

[6] K. Miyazaki, M. Yamamura, and S. Kobayashi, k-Certainty Exploration Method: an action selector to identify the environment in reinforcement learning, Artificial Intelligence, vol.91, no.1, pp.155-171, 1997.

[7] G. Kuhlmann, P. Stone, R. Mooney, and J. Shavlik, Guiding a reinforcement learner with natural language advice: Initial results in RoboCup soccer, The AAAI-2004 workshop on supervisory control of learning and adaptive systems, 2004.

[8] D.L. Moreno, C.V. Regueiro, R. Iglesias, and S. Barro, Using prior knowledge to improve reinforcement learning in mobile robotics, Proc. Towards Autonomous Robotics Systems. Univ. of Essex, UK, 2004.

[9] G. Brockman, V. Cheung, L. Pettersson, J. Schneider, J. Schulman, J. Tang, and W. Zaremba, OpenAI Gym., arXiv preprint arXiv: 1606.01540, 2016.

[10] L.X. Wang, A Course in Fuzzy Systems and Control, Prentice Hall, 1997.

[11] D. Li, D. Cheung, X. Shi, and V. Ng, Uncertainty reasoning based on cloud models in controllers, Computers \& Mathematics with Applications, vol.35, no.3, pp.99-123, 1998.

[12] D.Y Li and Y. Du. Artificial Intelligence with Uncertainty, National Defense Industry Press, Beijing, May 2014.

[13] T.P. Lillicrap, J.J. Hunt, A. Pritzel, N. Heess, T. Erez, Y. Tassa, D. Silver, and D. Wierstra, Continuous control with deep reinforcement learning, arXiv preprint arXiv:1509.02971, 2015. 